\title{
Foramen of Monroe
}

National Cancer Institute

\section{Source}

National Cancer Institute. Foramen of Monroe. NCI Thesaurus. Code C32627.

The connection between the lateral ventricles with the third ventricle, which allows for the flow of CSF between the structures. 